\title{
Air Entrapment Causing Inappropriate Shock From a Subcutaneous Implantable Cardioverter Defibrillator
}

\author{
Ying Chi Yanga, c, Thein Tun Aung ${ }^{\mathrm{b}}$, Steven J. Bailin ${ }^{\mathrm{b}}$, Troy E. Rhodes ${ }^{\mathrm{b}}$
}

\begin{abstract}
Subcutaneous implantable cardioverter defibrillator (S-ICD) is an accepted alternative to conventional transvenous devices. Their efficacy in arrhythmia management is comparable to ICDs. However, those devices also have limitations such as lack of anti-tachycardia pacing capability or higher occurrence of device oversensing associated with inappropriate shocks. Air entrapment inside one or more of subcutaneous pockets has been reported as one of uncommon causes of device malfunction. It is important to recognize the wandering or drifting baseline signals during device interrogation for timely diagnosis and appropriate treatment.
\end{abstract}

Keywords: Subcutaneous implantable cardioverter defibrillator; Air entrapment; Inappropriate shock

\section{Introduction}

Subcutaneous implantable cardioverter defibrillator (S-ICD) is a viable alternative to conventional ICD systems. The use of $\mathrm{S}-\mathrm{ICD}$ is more favorable in younger patients since it is associated with relatively lower lifetime risk of infection compared to transvenous devices. It can also be considered in individuals with challenging vascular access such as hemodialysis patients. However, S-ICD system has its own limitations. We present a case where an S-ICD delivered an inappropriate shock from oversensing due to air entrapment inside one of the subcutaneous pockets.

\section{Case Report}

A 58-year-old lady with a history of left-sided breast carcino-

Manuscript submitted February 9, 2019, accepted February 26, 2019

aDepartment of Internal Medicine, University of Iowa, Iowa City, IA, USA bDepartment of Cardiac Electrophysiology, University of Iowa, Iowa City, IA, USA

${ }^{c}$ Corresponding Author: Ying Chi Yang, Department of Internal Medicine, University of Iowa, 200 Hawkins Dr., Iowa City, IA 52241, USA.

Email: myomintun3@gmail.com

doi: https://doi.org/10.14740/cr848 ma status post left total mastectomy and chemotherapy underwent S-ICD implantation for anthraxcycline-induced cardiomyopathy with low ejection fraction. S-ICD was implanted with two-incision technique. The generator pocket was created by a vertical incision from left sixth to eighth intercostal spaces at the mid-axillary line. The substernal pocket was established by a second incision below the xiphoid process. After tunneling the defibrillator coil and connecting to the generator, the wounds were closed in three layers. Defibrillation threshold testing was successful at the first attempt with a $65 \mathrm{~J}$ shock. Tachytherapy settings were noted with shock zone at $220 \mathrm{bpm}$ and conditional shock zone at $200 \mathrm{bpm}$. All three vectors passed during the screening and the device was set to sense the primary vector. Chest X-rays confirmed the proper device and lead placement.

The patient was kept on cardiac telemetry for overnight observation. Few hours after the procedure, the patient received an inappropriate shock while sitting on the bed. She was asymptomatic and in sinus rhythm at the time of shock delivery as shown by telemetry. The device interrogation showed an inappropriate shock from oversensing (Fig. 1). There was no T-wave oversensing detected. The oversensing was not reproducible with any postural change or left arm isometric exercises.

Provocative manipulations to the defibrillator pocket or distal electrode did not reproduce the finding. However, ma-

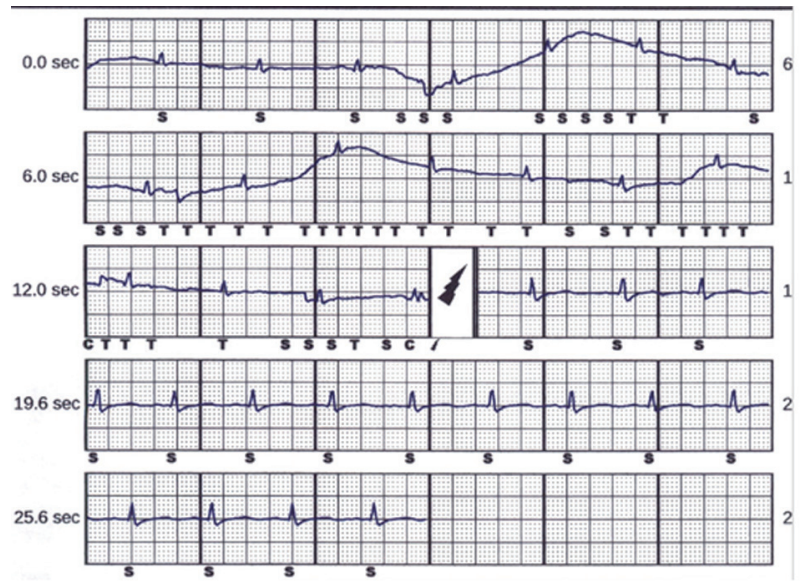

Figure 1. The tracing shows oversensing of the S-ICD with subsequent inappropriate shock. Note the wandering baseline starting from 2 to 14 s. The oversensing was also noted at the same time of device's inappropriate shock delivery. After the shock, sinus rhythm continued. 

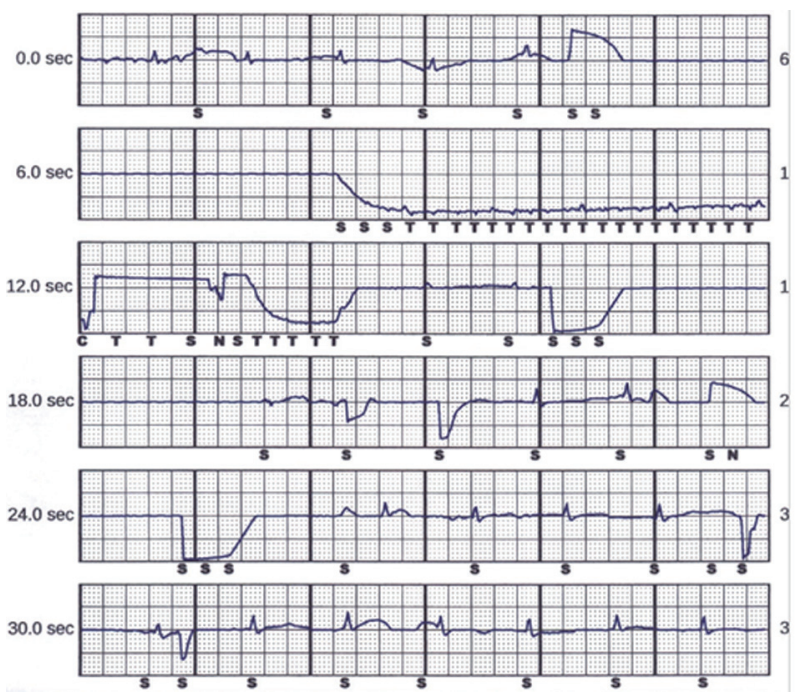

Figure 2. Tracing of the air entrapment in the S-ICD pocket causing abnormal sensing (from 8 to $12 \mathrm{~s}$ ) and wandering baseline pattern (from 4 to $27 \mathrm{~s}$ ).

nipulation over the substernal subcutaneous pocket reproduced the identical oversensing pattern (Fig. 2). After the device was reprogrammed to the secondary vector which is from the distal electrode to the generator, no more oversensing or inappropriate shock was noted (Fig. 3). Device interrogation at 2 weeks later during a follow-up visit did not show any more discharge.

\section{Discussion}

S-ICD has been accepted in the USA as one of the devicebased therapies since 2012. The efficacy of S-ICD in terminating ventricular tachyarrhythmias has been proven comparable to conventional ICD systems $[1,2]$. The use of S-ICD may be favorable in younger patient population as transvenous devices are associated with higher lifetime risk of infection [3-5]. It can also be considered in individuals with challenging vascular access such as hemodialysis patients. However, S-ICD system has its own limitations. It does not possess anti-tachycardia pacing capability and is associated with more inappropriate shocks compared to transvenous devices. The overall incidence of inappropriate shock varies in different studies ranging from $4-25 \%$, and inappropriate shocks are associated with increase in morbidity $[2,6,7]$. The majority of inappropriate shocks are related to abnormal sensing, particularly oversens-

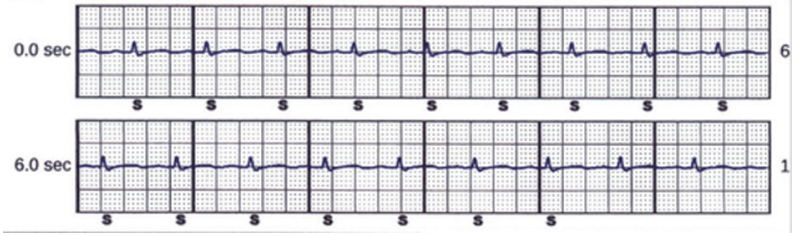

Figure 3. The tracing after reprogramming the device to sense a different vector. Only QRS complexes were sensed without any T wave oversensing at baseline.

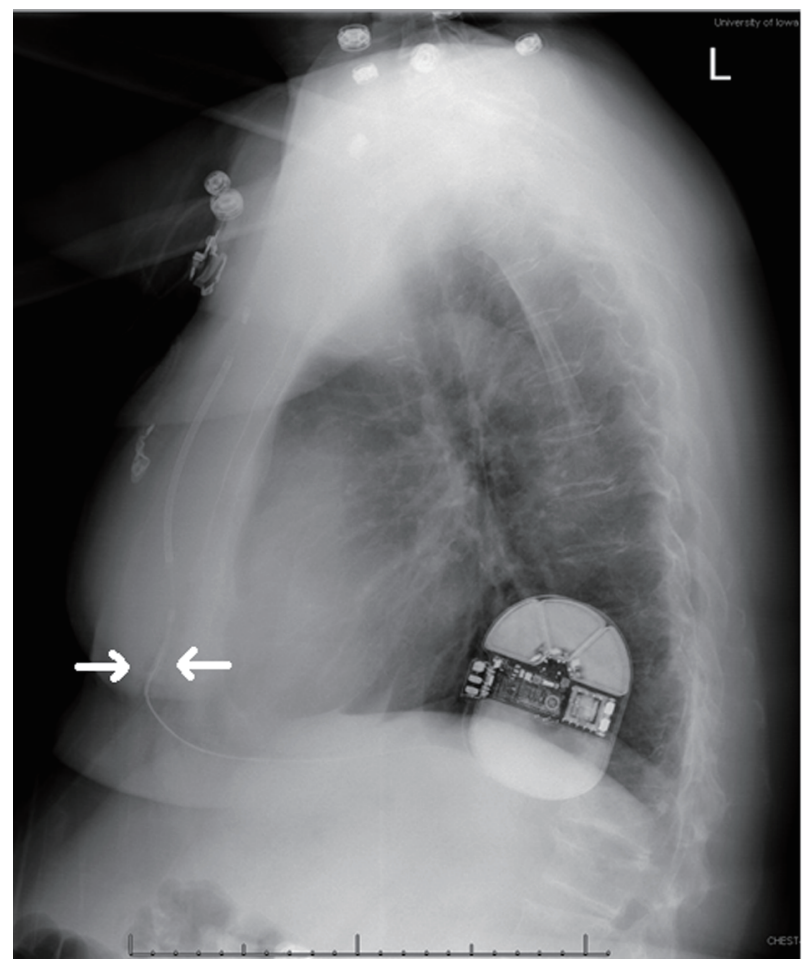

Figure 4. Radiolucency below the sternum (arrows) represents a small area of air entrapment within the substernal subcutaneous pocket.

ing. Unlike conventional devices, the most common cause of inappropriate shock by S-ICD is T-wave oversensing.

The manufacturer, Boston Scientific (Cambridge, MA, USA) suggested to ensure the absence of air entrapment within subcutaneous pockets during the implantation process to optimize device sensing and shock delivery. Flushing the sternal track with saline, massaging the skin over the lead and proper suturing proper suturing over the electrodes are suggested to minimize the air entrapment. Chest X-rays can often diagnose air entrapment when a large quantity of air sits inside a subcutaneous pocket. However, small pockets of air retention can sometimes be challenging. The lateral view of chest X-rays in our patient illustrated a small area of air collection inside substernal subcutaneous pocket (Fig. 4).

The hypothesis of air entrapment interfering with implantable cardiac devices was first established in 1979. Kreis et al reported a case of air entrapment as a cause of pacemaker dysfunction [8]. The first case of S-ICD's inappropriate shock from entrapped air inside the subcutaneous pocket was published in 2014 , and then it has been reported sporadically as a cause of inappropriate shocks $[9,10]$.

Two kinds of artifacts are commonly seen with the air entrapment. Both are related to the entrapped air insulating the sensing electrode. Firstly, sudden alternations in voltage due to the air movement inside the pocket result in sharp deflections. Those sharp deflections may be inappropriately sensed by the S-ICD as ventricular tachyarrhythmia. Second is a wandering or drifting baseline which can also be marked incorrectly by the S-ICD as ventricular tachyarrhythmia. The latter is believed to be the etiology of inappropriate shock in our case. 
The immediate step of managing the device malfunction from air entrapment is reprogramming the device to sense a different vector as entrapped air is often absorbed into tissues within few weeks. In our case, resetting the sensing vector from primary (sensing from the proximal electrode to the can) to secondary (sensing from the distal electrode to the can) terminated the further occurrence of abnormal sensing and inappropriate shock delivery.

\section{Conclusions}

Air entrapment is a rare but potential etiology of device oversensing and inappropriate shock delivery. It can be minimized with the application of a proper surgical technique during implantation. Air pockets are best visualized in the lateral view of chest X-rays. However, the diagnosis can sometimes be challenging. It is important to recognize the wandering or drifting baseline signals often found prior to the delivery of the inappropriate shock upon the device interrogation. The management is reprogramming the device to sense a different vector till the resolution of air entrapment.

\section{Acknowledgments}

None.

\section{Conflict of Interest}

Authors declare no conflict of interest.

\section{Financial Disclosure}

Authors declare no financial disclosure.

\section{Informed Consent}

Not applicable.

\section{Author's Contribution}

YCY is the corresponding/main author. TTA offered expert consultations to the case. SJB and TER are electrophysiologists who performed the procedures of the case and reviewed the manuscript.

\section{References}

1. Weiss R, Knight BP, Gold MR, Leon AR, Herre JM, Hood M, Rashtian M, et al. Safety and efficacy of a totally subcutaneous implantable-cardioverter defibrillator. Circulation. 2013;128(9):944-953.

2. Kobe J, Reinke F, Meyer C, Shin DI, Martens E, Kaab S, Loher A, et al. Implantation and follow-up of totally subcutaneous versus conventional implantable cardioverterdefibrillators: a multicenter case-control study. Heart Rhythm. 2013;10(1):29-36.

3. Wilkoff BL. How to treat and identify device infections. Heart Rhythm. 2007;4(11):1467-1470.

4. Le KY, Sohail MR, Friedman PA, Uslan DZ, Cha SS, Hayes DL, Wilson WR, et al. Clinical predictors of cardiovascular implantable electronic device-related infective endocarditis. Pacing Clin Electrophysiol. 2011;34(4):450459.

5. Eckstein J, Koller MT, Zabel M, Kalusche D, Schaer BA, Osswald S, Sticherling C. Necessity for surgical revision of defibrillator leads implanted long-term: causes and management. Circulation. 2008;117(21):2727-2733.

6. Jarman JW, Lascelles K, Wong T, Markides V, Clague JR, Till J. Clinical experience of entirely subcutaneous implantable cardioverter-defibrillators in children and adults: cause for caution. Eur Heart J. 2012;33(11):13511359.

7. Rosenqvist M, Beyer T, Block M, den Dulk K, Minten J, Lindemans F. Adverse events with transvenous implantable cardioverter-defibrillators: a prospective multicenter study. European 7219 Jewel ICD investigators. Circulation. 1998;98(7):663-670.

8. Kreis DJ, Jr., LiCalzi L, Shaw RK. Air entrapment as a cause of transient cardiac pacemaker malfunction. Pacing Clin Electrophysiol. 1979;2(6):641-644.

9. Zipse MM, Sauer WH, Varosy PD, Aleong RG, Nguyen DT. Inappropriate shocks due to subcutaneous air in a patient with a subcutaneous cardiac defibrillator. Circ Arrhythm Electrophysiol. 2014;7(4):768-770.

10. Yap SC, Bhagwandien RE, Szili-Torok T, Theuns D. Air entrapment causing early inappropriate shocks in a patient with a subcutaneous cardioverter-defibrillator. HeartRhythm Case Rep. 2015;1(3):156-158. 